\title{
Thymic tumours associated with Myasthenia gravis: a long term observation study of operated patients
}

\author{
Bak V, Spalek P, Petrovajova T, Vichova B, Oravsky M, Luha J, Schnorrer M \\ 3rd Clinic of Surgery, University Hospital, Bratislava, Slovakia. mrvbak@gmail.com
}

\begin{abstract}
Primary tumours of thymus, thymoma and thymic carcinoma, are very rare, they represent less than $1 \%$ of all neoplasms. The most common manifestation of thymoma with appearance of 40 to $50 \%$ of the neuromuscular autoimmune disease is myasthenia gravis (MG). The performance of a complete resection is the most predictive indicator for long-term survival of patients with thymic tumour, also important prognostic factors are the histological type of thymic tumour and its invasiveness. The aim of our study is to study the long-term survival of patients after the resection of the tumour, as well as enhanced efficiency of radical thymectomy in the complex treatment of myasthenia gravis. From 1989 to 2010 we operated on 369 patients with MG. Out of 49 patients $(13.7 \%) 38$ cases $(76.6 \%)$ were thymomas and 11 cases $(23.4 \%)$ thymic carcinomas. Complete removal of tumours (stage I, II, and III) were performed in 45 (92\%) cases. Of the 41 living patients (83.7 \%), three $(7.3 \%)$ were diagnosed with metastatic thymic carcinoma or thymoma, and treated with adjuvant therapy. In one case, the patient died due to generalization of the thymic carcinoma. Statistical analysis with KaplanMeier method showed better overall survival of patients with thymoma than patients with thymic carcinoma. The difference in survival curves was not significant. (Mantel-Cox $p=0.479$, Generalized Wilcoxon $p=0.326$ ). In terms of treatment of Myasthenia gravis associated with thymoma, we achieved $70 \%$ successful clinical and pharmacological remission. On the other hand, paraneoplastic MGAT has the worst prognosis of all the other forms of MG. The difference between MGAT to every other form of MG was statistically significant (Tab. 6, Fig. 1, Ref. 28). Full Text in PDF www.elis.sk.

Key words: thymoma and thymic carcinoma, myasthenia gravis, radical thymectomy, overall survival, clinical and pharmacological remission.
\end{abstract}

Thymomas with thymic carcinomas present primary tumours of thymus having indolent course and sporadic occurrence. Generally, they account for less than $1 \%$ of all oncological neoplasms but comprise $20-25 \%$ of all mediastinal tumours (1). A larger body of literature now provides further insight into biological behaviour of thymoma playing a role in the pathogenesis of myasthenia gravis (MG). Therefore, myasthenia gravis associated with thymomas (MGAT), is also called paraneoplastic, which is an organspecific autoimmune disorder characterised by wavering muscular weakness and abnormal fatigue. In imunopathogenesis of MGAT participate thymic and extrathymic mechanisms. Henceforth, radical surgical removal of thymoma does not always lead to clinical improvement of MG and decreasing titre of autoantibodies that block acetylcholine receptors (AChR) (2).

Thymomas are characterised by their variability of morphological appearance and are often associated with various autoimmune diseases. Previous studies report the correlation between their morphological appearance and clinical behaviour. Based on

3rd Clinic of Surgery, University Hospital, Bratislava, and Centre for Neuromuscular disorders of Neurological clinic, University Hospital Bratislava Ruzinov, Slovakia

Address for correspondence: V. Bak, MD, 3rd Clinic of Surgery, University Hospital, SNP 10, SK-814 65 Bratislava, Slovakia.

Phone: +421.2 .57887441$ the facts, the last histological classification was presented by the WHO in 1999 which was slightly modified in 2004 showing a relation to clinical behaviour, tumour staging system, the prognosis and the association with $\mathrm{MG}(3,6,7)$ (Tab. 1).

According to the WHO classification, epithelial tumours are divided in two main groups. Thymomas are characterised as organotypic thymus-like tumours classified in 5 types (A,AB, B1, B2 and B3), taking into account the morphology of epithelial cells as well as the ratio of epithelial cells to lymphocytes $(4,5,6,7)$. Thymic carcinomas are described as epithelial malignancies, their appearance resembles tumours originating from the other organs and they have no capsule and infiltrate surrounding structures. The most common subtypes of thymic carcinomas are: squamocellular, basaloid, mucoepidermoid, lymphoepithelioma-like, clear-cell, undifferentiated/anaplastic and group neuroendocrine tumours $(8,9)$.

Besides determination of histological type of thymoma, the staging system has prognostic value as well. A TNM staging system that has gained widespread acceptance was proposed by Masaoka and his colleagues in 1981 (Tab. 2). It is based on the extent of either macroscopic or microscopic invasion into mediastinal structures. Peri-operative determination of tumour extent followed by radical or palliative surgical removal is considered as main prognostic factor (10).

The sex distribution of patients with thymic tumours is roughly equal in the broad age, with a peak around 40 to 60 years of age 
Tab. 1. Characteristics of thymic tumours WHO 2004.

\begin{tabular}{lccccc}
\hline Type & A & AB & B1 & B2 & B3 \\
Shape of cells & Spindle & Spindle/Polygonal & Polygonal & Polygonal & Polygonal \\
Atypia of cells & Minimum & Minimum & Minimum & Mild & Moderate \\
Volume of lymphocytes & Mild & Moderate & High & Moderate & High \\
Organotypia & Yes & Yes & Yes & Yes & Mild \\
\hline
\end{tabular}

Tab. 2. Masaoka Staging System.

\begin{tabular}{clc}
\hline Stage & Definition & Occurrence \\
\hline I & $\begin{array}{l}\text { Macroscopically encapsulated tumour, with no } \\
\text { microscopic capsular invasion }\end{array}$ & $35-50 \%$ \\
II & $\begin{array}{l}\text { Macroscopic or microscopic invasion into cap- } \\
\text { sule and surrounding fatty tissue or mediastinal } \\
\text { pleura }\end{array}$ & $15-25 \%$ \\
III & $\begin{array}{l}\text { Macroscopic invasion into neighbouring organs } \\
\text { IV A }\end{array}$ & $\begin{array}{l}\text { Pleural or pericardial metastases } \\
\text { IV B }\end{array}$ \\
\hline
\end{tabular}

$(11,12)$. Thymomas are associated with several parathymic syndromes which is an autoimmune condition, most commonly with MG occurring in 40 to $50 \%$ of patients $(13,14)$. Conversely, approximately 10 to $15 \%$ of patients with MG are found to have thymoma $(15,16,17)$. Paraneoplastic MG is a seropositive form with antibodies against $\mathrm{AChR}$ leading to the diagnosis of thymoma, which is asymptomatic because of slow growth and even to a farther reaching extent. Occasionally, it may be accidentally found as mediastinal mass by computer tomography. Those with local symptoms related to the intrathoracic mass may have chest pain, cough, afterwards dyspnoea, haemoptysis, stridor and dysphonia. Superior vena cava syndrome, Horner's syndrome, arrhythmias and weight loss occur rarely with more aggressive tumours. By the infiltration of oesophagus they may cause dysphagia with odynophagia. Thymic tumours rarely metastasize affecting intrathoracic structures as pericardium, pleura and diaphragm or the distant sites $(18,18,20)$.

Surgical treatment is the mainstay of treatment of thymomas, because the vast majority (90-95\%) of these tumours are localized. Radical operation with removing the adjacent fatty tissue has shown to be the most significant prognostic factor for the overall survival $(21,22)$. The 10 -year overall survival rate after complete resection is $88 \%, 70 \%, 57 \%$ and $38 \%$ for I, II, III, and IV stage respectively (23). The best measure of outcome after resection may be the recurrence rate that occurs most likely after the resection of extensive tumours, because it is largely dependent on its invasion. An average of $80 \%$ of all recurrences are local and they should be completely resected whenever it is possible and adjuvant therapy should be administered. This approach is associated with good long-term survival $(13,24,25)$.

\section{Patients and methods}

A retrospective review of all patients undergoing resection for thymic tumours associated with MG was performed at a single centre between January 1989 and December 2010. During this time period, 369 patients underwent surgery as the treatment of MG.
Of the total operated patients 49 were selected with post-operative findings of thymic tumours.

All patients were diagnosed with MG by the neurologist in Myasthenia centre for Neuromuscular disorders at Bratislava University Hospital. The diagnosis was established based on neurological a myological investigation, set of titre of antibodies (antibodies against $\mathrm{AChR}$ and muscular-specific tyrosine-kinase, antistriatal antibodies) and using electrophysiological investigation EMG (repetitive stimulation, single fibre electromyography). Each patient underwent radiology to confirm or alternatively exclude the presence of thymic tumour. Initially, X-ray imaging was used having very low sensitivity, only visualising gross masses of tumours. Performance of computed tomography has markedly given more precise details of thymic tumours, their relation to great vessels and surrounding structures. A vast majority of patients with MGAT was administered immunosuppresive therapy preoperatively, in combination with plasmapheresis and intravenous imunoglobine in some cases. After achieving a decrease of clinical symptomatology of MG whenever most of the patients were asymptomatic having mantained low-doses of imunosupressive therapy, then the operation was indicated. The preoperative therapy is necessary due to tolerance of general anesthesia, the course of operation and avoidance of deterioration of $\mathrm{MG}$.

We preferred performing thymectomy, an upper partial sternotomy, for the advantage of good exposure. Through a 10 to 12 $\mathrm{cm}$ skin incision, with the sternum retracted, adequate visualization of thymus and its cervical extensions is obtained for performance of total radical thymectomy that means removing of whole thymus and adjacent fatty tissue from anterior mediastinum. Once the thymus is dissected completely, the pericardium, ascendent aorta, anonyma vein and both pleura are visualized. By the macroscopic findings of thymic tumours the effort was highly made to undertake more extensive operation and complete resection. The sternum is then sutured using three metal wires and having a chest tube placed retrosternally for adequate postoperative drainage of mediastinum.

Upon completion of the operation, all patients were followed up by a neurologist in myasthenic centre for neuromuscular disorders. In case of histological findings of thymic tumour the assessment of administration of adjuvant therapy was made by an oncologist. Histological findings were initially classified according to former morphological system proposed by Bernatz then to the Muller-Hermelink system and in recent years WHO system. Due to un-unified classification system in the past 20 years, we were not able to distribute patients according to the newest classification system. Therefore, we selected the patients into two groups in accordance with histological type, thymoma or thymic carcinoma. Based on the results of surgical treatment we evaluated the efficiency of radical thymectomy and survival period in 
individual groups of patients. In terms of treatment of MG different improvement and remission of symptoms have been achieved and evaluated in the mentioned groups of patients. The aim of treatment of MG was to accomplish clinical or pharmacological remission that means no symptom without or with low-doses of maintenance therapy of immunosuppressant, not having any corticosteroids in therapy.

All results were analysed by software, statistical package SPSS version 15.0. For the description of data file the frequency tables were used as well as basic characteristics like mean time, mean age, median and range. The efficiency of radical thymectomy was assessed, based on the count of the complete resection in the different stages of tumours classified by Masaoka. Statistical analysis was focused on the evaluation of the survival period with groups of patients having thymoma or thymic carcinoma. Groups of patients sorted by the mentioned criteria were evaluated with Kaplan-Meier method. The differences in survival curves were tested with Mantel-Cox test and generalized Wilcoxon test. The accomplishment of clinical and pharmacological remission in terms of treatment of MG was assessed by certain percentage in the different pathogenetic forms of MG. The Pearson Chi-Square test was used to analyse the difference between three different pathogenetic forms of MG. The level of statistical significance was set at $\mathrm{p}=0.05$.

\section{Results}

Radical thymectomy for MG was performed in 369 patients and 49 of them (13.3\%) had histology of thymic tumours. Thymic tumours distribution of patients were $38(76.6 \%)$ thymomas and $11(23.4 \%)$ thymic carcinomas. The baseline characteristics of patients are shown in Table 3.

The complete resection of tumours (stage I, II, and III) was achieved in $45(92 \%)$ cases and only in $4(8 \%)$ patients with stage III and IVa a subtotal resection (debulking) was performed because of advance state of thymic carcinoma and infiltration of great vessels and pericardium. However, thymic tumours in stage III were completely resected in $57.1 \%$. The occurrence of thymic tumours in accordance with their stage of invasion proposed by Masaoka system are shown in Table 4. Thymic tumours of stage I and II occurred in $41(83.7 \%)$ patients that is a considerable result in terms of overall survival. The rest of the patients $(16.7 \%)$ diagnosed with stage III and IVa received adjuvant radiotherapy.

In one case we decided to re-operate. It was a local recurrence of thymic tumour diagnosed 10 years after complete resection of thymic carcinoma classified in stage III. The diagnosis was made by
Tab. 4. Distribution of patients classified by Masaoka system.

\begin{tabular}{lccc}
\hline $\begin{array}{l}\text { Tumour stage } \\
\text { of Masaoka } \\
\text { classification }\end{array}$ & Thymoma & $\begin{array}{c}\text { Thymic } \\
\text { carcinoma }\end{array}$ & Total \\
\cline { 2 - 4 } & 24 & 0 & 24 \\
I & 13 & 4 & 17 \\
II & 0 & 7 & 7 \\
III & 0 & 1 & 1 \\
IVa & & & \\
\hline
\end{tabular}

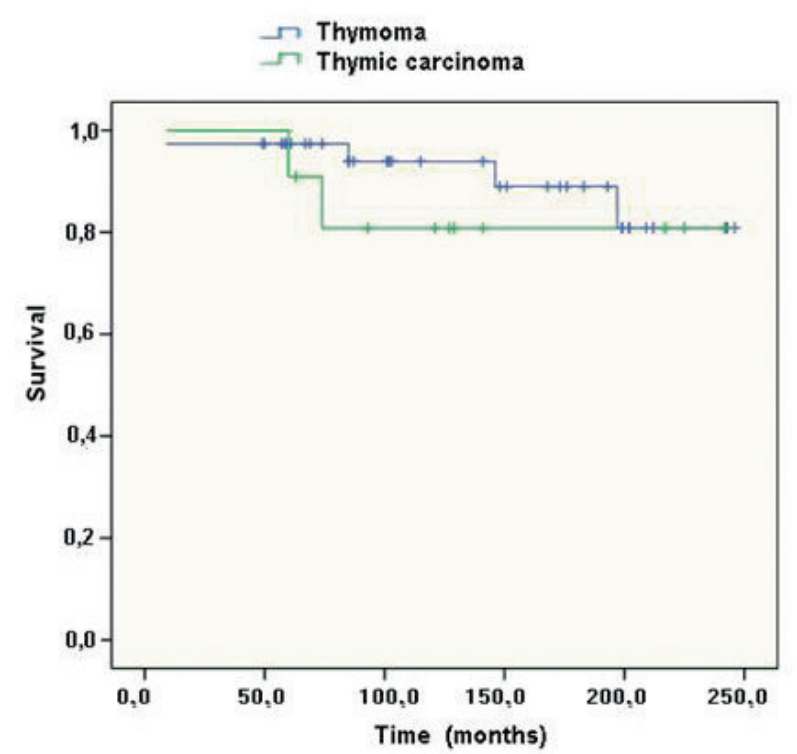

Fig. 1. Overall survival of patients with thymoma or thymic carcinoma after thymectomy and following immunosuppressive therapy shows better overall survival of patients with thymoma than patients with thymic carcinoma (Mantel-Cox $p=0.479$, Generalized Wilcoxon $p=0.326$ ).

performance of PET-CT and local metastases of both lungs were also found. We decided on the removal of the local recurrence but only biopsy could be performed resulting in confirmation for local recurrence of thymic carcinoma. Then the patient received chemotherapy.

With a mean time of 9.9 years, (min.2.3 - max 20.2 years), a follow up of 41 patients $(83.7 \%$ ) resulted in disease-free survival. Two of the patients are unknown and five died with no relation to the underlying disease. The cause of death for three patients were heart failure prior to heart attack, one progression of lung fibrosis of chronic obstructive lung disease and one prior to trauma accident. Only one case of death was due to generalised disseminated thymic carcinoma, in spite of the use of all three common therapeutic modalities which included surgery, radiation and chemotherapy. In long term follow-up of patients, three of them were diagnosed

Tab. 3. Baseline characteristics of patients.

\begin{tabular}{lcc}
\hline Characteristics & Patients with thymoma & Patients with thymic carcinoma \\
\hline Sex - Male / Female & $8 / 30$ & $3 / 8$ \\
Age - Median (min. - max.), years & $42($ min. $18-\max .69)$ & $45(\min .33-$ max.68) \\
Mean time of history, months & $9+/-4$ & $6+/-3$ \\
Mean time of preoperative treatment, months & $11+/-5$ & $9+/-4$ \\
Mean time of history and preoperative treatment, months & $20+/-10$ months \\
Mean time of staying at hospital & $9+/-3,5$ & $14+/-8$ months \\
\hline
\end{tabular}


Tab. 5. Result of treatment of MGAT.

\begin{tabular}{|c|c|c|c|}
\hline & & Thymoma & Thymic carcinoma \\
\hline \multirow{4}{*}{ 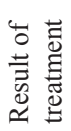 } & CR & $68,8 \%$ & $31,3 \%$ \\
\hline & PR & $88,2 \%$ & $11,8 \%$ \\
\hline & SI & $83,3 \%$ & $16,7 \%$ \\
\hline & MI & $0 \%$ & $100 \%$ \\
\hline
\end{tabular}

Abbreviations: CR - Clinical remission, PR - Pharmacological remision, SI - Significant improvement, $\mathrm{MI}$ - Mild improvement

with intrathoracic metastases of confirmed thymic carcinoma and thymoma B3. After this diagnosis they received chemotherapy with an objective average response.

The overall survival of observing groups with mean time of 10 years with follow-up is remarkably well. Statistical analysis with Kaplan-Meier method (Fig. 1) shows better overall survival of patients with thymoma than patients with thymic carcinoma. The difference in survival curves was not significant. (Mantel-Cox $\mathrm{p}=0.479$, Generalized Wilcoxon $\mathrm{p}=0.326$ ).

Concerning the treatment of MG in the observed 47 patients, the clinical remission in 16 patients $(34 \%)$ and pharmacological remission in 17 patients (36\%) were achieved. The achievement of successfuly treated patients were $70 \%$ of all. Patients in clinical and pharmacological remission were mostly with thymoma in $68.8 \%$ and $88.8 \%$ respectively than with thymic carcinoma in $31.2 \%$ and $11.8 \%$ respectively (Tab. 5). The mild improvement of MG symptomatology has been found in patients with thymic carcinoma. Statistical evaluation showed significant difference between the mentioned groups (Chi-Square test $\mathrm{p}=0.034$ ). On the other hand, the paraneoplastic MG has the worst prognosis in comparison to other different forms of MG. The outcome of treated patients with different forms of MG is shown in Table 6. The highest percentage of achievement in the clinical remission was seen with seropositive MG without thymic tumour, but the lowest percentage in patients having MGAT with a statistical difference (Chi-Square test $\mathrm{p}=0.015$ ).

\section{Discussion}

The complex treatment of thymic tumour associated with paraneoplastic MG has positive progression. In the era before immu- nosuppressive therapy thymectomy reached $50 \%$ mortality rate in the early postoperative period, usually because of respiratory failure. The substantial cause of the unfavourable outcome was a conservative attitude for surgery as well. The indication for surgery was set in the late stage of MG $(26,20)$. The main change in the pathogenetic therapy and early indication for surgery connected with the immunosuppressive preoperative therapy caused thymectomy as operative intervention with minimal mortality $(2.5 \%)$ and morbidity ( $4.5 \%$ ). In our 20-years experience of performing radical transsternal thymectomy there was one case with postoperative death. Thymectomy was performed as the last option after exhausting all conservative treatment for a patient in myasthenic crisis to ameliorate health condition. Any other consequential complication was not remarked with postoperative morbidity $9.5 \%$.

In terms of surgery and oncology, thymic tumours became the absolute indication for operative treatment. The higher age of a patient or serious co-morbidity might be contraindication for surgery. The Medial sternal split is the most common approach to the anterior mediastinum. The size of performed sternotomy, partial or total, is based on the surgeon's decision and local findings.

Long discussed prognostic factor is the histological type of thymoma. Currently, there are claims of the correlation between different types of tumours and their invasiveness. Patients with thymoma type $\mathrm{A}, \mathrm{AB}, \mathrm{B} 1$ have a significantly longer survival rate than patients with a thymoma type B2 or B3. Several proofs show the worse prognosis for patients with thymic tumours $(6,7)$. In our series there is still a small number of patients classified according to the latest WHO classification. Until now it is recorded that the patient's death was just the generalization of the thymic carcinoma. Three other patients with intrathoracic metastases have verified thymic carcinoma and thymoma B3. This is in accordance with the results of several studies of a poor prognosis of the mentioned types of thymoma, as well as, the very fact of correlated classified tumours by the WHO.

It is becoming clearer that the pathogenesis of MG patients with thymoma differs from other types of MG pathogenesis. The main functional difference is the presence of intratumorous production of autoantibodies. The mere initiation of autoimmune mechanism is in thymoma but the continuation of their activation of im-

Tab. 6. Result of treatment of pathogenic forms of Myasthenia gravis.

\begin{tabular}{|c|c|c|c|c|c|c|}
\hline & & & \multicolumn{3}{|c|}{ Pathogenetic forms of MG } & \multirow[b]{2}{*}{ Total } \\
\hline & & & $\begin{array}{c}\text { SPMG without } \\
\text { thymoma }\end{array}$ & SNMG & $\begin{array}{l}\text { SPMG with } \\
\text { thymoma }\end{array}$ & \\
\hline \multirow[t]{7}{*}{ Result of treatment } & CR & Count & 90 & 58 & 16 & 164 \\
\hline & & $\%$ within pathogenetic form & $55,6 \%$ & $43,3 \%$ & $34,0 \%$ & $47,8 \%$ \\
\hline & PR & Count & 35 & 35 & 17 & 87 \\
\hline & & $\%$ within pathogenetic form & $21,6 \%$ & $26,1 \%$ & $36,2 \%$ & $25,4 \%$ \\
\hline & & $\%$ within pathogenetic form & $19,8 \%$ & $26,1 \%$ & $25,5 \%$ & $23,0 \%$ \\
\hline & MI & Count & 5 & 6 & 2 & 13 \\
\hline & & $\%$ within pathogenetic form & $3,1 \%$ & $4,5 \%$ & $4,3 \%$ & $3,8 \%$ \\
\hline \multirow[t]{2}{*}{ Total } & & Count & 162 & 134 & 47 & 343 \\
\hline & & $\%$ within pathogenetic forms & $47,2 \%$ & $39,1 \%$ & $13,7 \%$ & $100,0 \%$ \\
\hline
\end{tabular}

Abbreviations: CR - Clinical remission, PR - Pharmacological remision, SI - Significant improvement, MI - Mild improvement, SPMG - seropositive form of myasthenia gravis, SNMG - seronegative form of myasthenia gravis 
munocompetent cells which produce autoantibodies are possible in places of retained residual thymus outside of the tumour. There are also assumptions that the activation of autoantibodies may take place in the non-thymatous immune system such as; lymph nodes, spleen and bone marrow. That would explain why thymectomy does not always decrease the titre of autoantibodies, while in some cases it improves clinical symptomatology $\operatorname{MG}(18,27,28)$. The effectiveness of thymectomy in the treatment of MGAT is still in question and the literature is uncertain. In accordance to our evaluation of the success of clinical and pharmacological remission the results were outstanding. Therefore, we consider thymectomy in the treatment of MGAT to be essential and effective.

Patients with early diagnosis of thymoma associated with MG, individually adjusted to preoperative immunosuppressive therapy followed by radical thymectomy, achieved remarkable results in long-term survival and contributed significantly in the treatment of paraneoplastic MG.

\section{References}

1. Pit'ha $\mathbf{J}$ et al. Klasifikace a biologie thymomu ve vztahu k myasthenia gravis. In: Otčenášková J, Pátková V, editors. Myasthenia Gravis, Prague, Maxdorf 2010: 217.

2. Špalek P, Schnorrer M, Krajč T. Diagnostika a liečba paraneoplastickej myasténie gravis asociovanej s tymómom. Neurológia 2010; 5 (2): 79-85.

3. Travis WD, Brambilla E, Müller-Hermelink HK, Harris CC. WHO Classification of Tumours: Pathology and Genetics of Tumours of the Lung, Pleura, Thymus and Heart. Oxford: Oxford University Press Inc: 2004.

4. Kondo K, Yoshizawa K, Tsuyuguchi M, Kimura S et al. WHO histologic classification is a prognostic indicator in thymoma. Ann Thorac Surg 2004; 77: 1183-1188.

5. Ströbel P, Bauer A, Puppe B, Kraushaar T et al. Tumor recurrence and survival in patients treated for thymomas and thymic squamous cell carcinomas: A retrospective analysis. J Clin Oncol 2004; 22: 1501-1509.

6. Okumura M, Shiono H, Minami M et al. Clinical and pathological aspects of thymic epithelial tumors. Gen Thrac Cardiovasc Surg 2008; 56: $10-16$.

7. Ströbel P, Marx A, Zetil A, Müller-Hermelink HK. Thymoma and Thymic Carcinoma . An Update of the WHO Classification 2004. Surg Today 2005; 35: 805-811.

8. Suster S, Rosai J. Thymic carcinoma: A clinicopathologic study of 60 cases. Cancer 1991; 67: 1025-1032.

9. Rosai J, Sobin L. Histological typing of tumours of the thymus, 2nd ed. In: World health Organization, International Histological Classification of Tumours. New York, Springer, 1999: 9-14.

10. Yamakawa Y, Masaoka A, Hashimoto $T$ et al. A tentative tumornode-metastasis classification of thymoma. Cancer 1991; 68: 1984-1987.
11. Vernino S. Paraneoplastic disorders affecting the neuromuscular junction or anterior horn cell. In: Myasthenia gravis and ALS. Continuum 2009; 15: 132-146.

12. Romi F, Gilhus NE, Varhaug JE et al. Disease severity and outcome in thymoma myasthenia gravis: a long term observation study. Eur J Neurol 2003; 10: 701-706.

13. Okumura M, Miyoshi S, Fujii Y et al. Recurrence of surgical treatment of thymomas with special reference to the involved organs. J Thorac Cardiovasc Surg 1999; 117: 605-613.

14. Kondo K, Monden Y. Therapy for thymic epithelial tumors: a clinical study of 1,320 patients from Japan. Ann Thrac Surg 2003; 76: 878-884.

15. Spalek P, Schnorrer M, Krajč T. Imunopatogenesis paraneoplastickej myasténie gravis asociovanej s tymómom. Neurológia 2010; 5: 7-11.

16. Levi N, Abramsky O, Lossos A et al. Extrathymic malignancies in patients with myasthenia Gravis. J Neurol Sci 2005.

17. Meriggioli MN, Sanders DB. Autoimmune myasthenia gravis: emerging clinical and biological heterogeneity. Lancet neurology 2009; 8: 475490.

18. Špalek P. Tymómy a paraneoplastická autoimunita. Cesk Neurol Neurochir 2002; 65/98: 367-373.

19. Riedel RF, Burfeind WR, Jr. Thymoma: benign appearance, malignant potential. Oncologist 2006; 11: 887-894.

20. Evoli A, Minicuci GM, Vitaliani R et al. Paraneoplastic disease associated with thymoma. J Neurol 2007; 254: 756-762.

21. Kondo Y, Monden Y. Thymoma and myasthenia gravis: a clinical study of 1,089 patients from Japan. Ann Thorac Surg 2005; 79: 219-224.

22. Schnützner J, Tvrdoň J. Chirurgická léčba thymomů. In: Schnützner J, Šmat V et al., editors. Myasthenia gravis - komplexní pojetí a chirurgická léčba. Praha: Galen; 2005: 63-71.

23. Detterbeck FC, Parsons AM. Thymic Tumors. Ann Thorac Surg 2004; 77: 1860-1869.

24. Wilkins KB, Sheikh E, Green R et al. Clinical and pathologic predictors of survival in patients with thymoma. Ann Surg 1999; 230: 562-574.

25. Ruffini E, Mancuso M, Oliaro A et al. Recurrence of thymoma: analysis of clinicopathologic features, treatment, and outcome. J Thorac cardiovasc Surg 1997; 113: 55-63.

26. Kac F, Yerdelen D, Sarica Y. Myasthenia gravis and invasive thymoma with multiple intracranial metastases. J Clin Neuromusc Dis 2003; 4: 171-173.

27. Ströbel P, Chuang WY, Marx A. Thymoma-associated paraneoplastic myasthenia gravis. In: Kaminski HJ, editor. Myasthenia gravis and related disorders. New York: Humana Press; 2009: 105-117.

28. Sommer N, Tackenberg N, Hohlfeld R. The immunopathogenesis of myasthenia gravis, In: Engel AG, editor. Neuromuscular junction disorders. Handbook of Clinical Neurology. Vol. 93 (3rd series). Amsterdam: Elsevier; 2008: 169-212. 